\title{
Analyze the Dose Results Calculated with AAA Algorithm in Eclipse Software of New Treatment Planning System
}

\author{
Soai DANG QUOC ${ }^{1}$, Quang BUI VINH ${ }^{2}$, Cuong BUI XUAN ${ }^{1}$, Toan HOANG VAN ${ }^{1}$ and Truong VU ${ }^{1}$ \\ 1. Medicinal Supplies, Medical Physics Division, Hanoi Oncology Hospital, 42A Thanh Nhan Street, Hai Ba Trung District, Hanoi \\ 100000, Vietnam
}

2. Department of Radiotherapy, Hanoi Oncology Hospital, 42A Thanh Nhan Street, Hai Ba Trung District, Hanoi 100000, Vietnam

\begin{abstract}
In this paper, the authors investigate the differences between the dose results calculated with AAA (Anisotropic Analytical Algorithm) algorithm in Eclipse 13.0 treatment planning system, which has just commissioned, with the measured doses. The AAA algorithm in the Eclipse software is used to calculate the absolute dose, relative dose in profile line, and percent depth dose of some specific field size in Blue Phantom. The calculated dose results were compared with measured dose based on Technical Reports Series No. 430 of International Atomic Energy Agency (IAEA No. 430). The authors have used CC13 ion chamber, Famer Chamber, water phantom, and Omnipro software to measure relative, absolute doses of these fields size. The AAA algorithm was also used to calculate the doses in IMRT (Intensity Modulated Radiotherapy) plan of fifty head and neck cancer patients. These IMRT plans were controlled quality in some points with plastic phantom, and famer chamber FC65. The differences between calculated dose, and measured doses are in the limit of IAEA 430 report. The results of the QA IMRT plan processing are fitting with ESTRO (European Society Therapeutic Radiation Oncology) Booklet No. 9.
\end{abstract}

Key words: Analytical anisotropic algorithm, dosimetry, relative dose, absolute dose.

\section{Introduction}

Ensuring the accuracy of the TPS (Treatment Planning System) based on the IAEA standard is a part of ensuring the optimal therapeutic dose for the tumor, limiting the malignant dose to healthy organs around the tumor, and ensuring radiotherapy safety for the treated patients with radiation therapy [1-5]. For the purpose of calibrating the dose calculation algorithm in a new radiotherapy TPS with using the experimental measurement method, many organizations from different countries, as well as the IAEA have conducted research and set standards for the accuracy of dose results calculated with the new TPS and measured dose results [1-3]. In this paper our group

Corresponding author: Soai DANG QUOC, M.Sc., research fields: radiotherapy, radiation safety, radiation protection. Email: dangquocsoai@gmail.com. presents the way and its results, which is used to control new TPS in our center.

\section{Materials and Methods}

The object of this study is a new Eclipse TPS, which has just commissioned in our hospital. Firstly the TPS is used to calculate absolute and relative dose for some special field in water phantom for example: reference field, rectangular field, non symmetric field, field with different SSD (Source to Skin Distance). The calculated doses are compared with measured dose in Blue Phantom.

The TPS is also used to plan for fifty head and neck cancer patients. All CT images of the patients, which have not been scattering due to metal replacement parts, are contoured all PTV (planning target volumes), spinal cord, right submandibular gland (DHP), left submadibular gland (DHT), right parotid gland (MTP), 
left parotid gland (MTT). A famer Chamber FC65 and plastic phantom are used to measured at all center points of PTV, DHP, DHT, MTP, MTT.

\subsection{Analyzation of Profiles and PDD (Percentage} Depth Dose)

The following criteria for $\delta$ are proposed for different regions in the beam as Figure. 1.

$$
\delta=100 \% \times \frac{D_{\text {calc }}-D_{\text {meas }}}{D_{\text {meas }}}
$$

where $D_{\text {calc }}$ is the calculated dose and $D_{\text {meas }}$ is the measured dose.

$\delta_{1}$ : for data points on the central beam axis beyond the depth of $d_{\max }$, that is the high dose and low dose gradient region. These points may range from $7 \%$ up to $100 \%$. The tolerance value for $\delta_{1}$ is $2 \%$ [1-3].

$\delta_{2}$ : for data points in the build-up region, in the penumbra, and in regions close to interfaces of inhomogeneities, these are the high dose high dose gradient regions. This criterion can be applied in the area between the phantom surface and the depth of the $90 \%$ isodose surface, as well as in the penumbra region. As an alternative, the shift of isodose lines (in mm) can be used. The dose gradient in these areas is generally larger than $3 \%$ per $\mathrm{mm}$. The tolerance value for $\delta_{2}$ is $10 \%$ or $2 \mathrm{~mm}[1,2]$.

$\delta_{3}$ : for data points not on the central beam axis beyond $d_{\max }$, that again is a high dose and low dose gradient area. The tolerance value for $\delta_{3}$ is $3 \%[1,2]$.

$\delta_{4}$ : for data points outside the geometrical beam edges and below shielding blocks, generally beyond $d_{\max }$, that is the area with a low dose and low dose gradient, for instance below $7 \%$ of the applied dose. The criterion $\delta_{4}$ is applied in areas of low dose, where the calculations are often inherently less accurate and where accuracy is often clinically less critical. Therefore in such cases it is not meaningful to relate deviations between calculations and measurements to the value of the locally measured dose alone. An appropriate alternative is to replace expression in those cases by:

$$
\delta_{4}=100 \% \times \frac{D_{\text {calc }}-D_{\text {meas }}}{D_{\text {meas } . c a x}}
$$

In this equation, for points outside the beam the deviation is normalized to the dose at a point at the same depth on the central beam axis (CAX). The tolerance value for $\delta_{4}$ is $3 \%[1,2]$.

\subsection{Absolute Dose Calculation}

The corrected measurement value of each field is calculated with the following formula $[3,8]$.

$$
M_{Q}=k_{T P} k_{\text {elec }} k_{\text {pol }} k_{s} \frac{\sum_{i=1}^{i=5} M_{i}}{5}
$$

where: $M_{i}$ : measured value of the field $[\mathrm{nC}] ; k_{\mathrm{TP}}$ : pressure and temperature correct factor; $k_{\text {elect }}$ : electrometer calibration factor; $k_{\mathrm{pol}}$ : polarity correction factor; $k s$ : recombination correction factor.
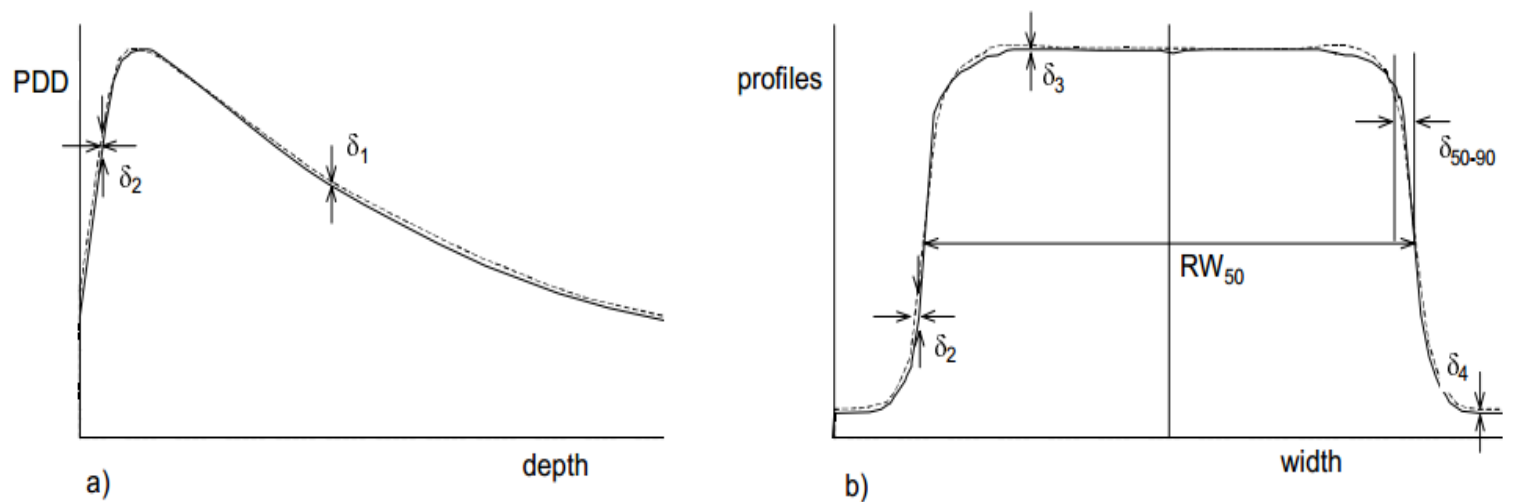

Fig. 1 Position of $\delta_{1}-\delta_{4}$ to compare calculated (dashed line) and measured (drawn line) dose values of a PDD curve (a) and a profile (b). 
The absolute dose of reference field size is calculated with the formula $[4,5,8]$ :

$$
D_{w, Q_{-} r e f}=M_{Q_{-} r e f} N_{D, w, Q_{0}} k_{Q, Q_{0}}
$$

where: $\mathrm{M}_{\mathrm{Q} \_ \text {ref: }}$ c corrected measurement value of reference field;

The absolute dose of other field size is calculated with formula:

$$
D_{w, Q}=k_{d c o} M_{Q} N_{D, w, Q_{0}} k_{Q, Q_{0}}
$$

where:

$k_{d c o}$ $=\frac{100 c G y}{D_{w . Q_{-} \text {ref }}}$ this is daily corrected output of LINAC.

According to the task group 53 and IAEA technical reports series No. 430 [1], the difference between the calculated dose and the measured dose has tolerance as Table 1.

\subsection{Analyzation for IMRT Plan Results}

The measured results in IMRT plans are subtracted the background radiation, corrected as the following equation.

$$
D_{f}=\left(D_{m}-D_{b}\right) k_{T P} k_{\text {elec }} k_{p o l} k_{s}
$$

where: $D_{\mathrm{m}}$ : measured dose [cGy], $D_{\mathrm{b}}$ : backgound [cGy], $k_{\text {elec }}=1$ is electrometer calibration factor, $k_{\text {pol }}=$
1.0015643 polarity correction factor, $k_{s}$ : recombination correction factor.

\section{Results}

If the relative dose difference is larger than the tolerance values, the authors will recheck method, set-up and equipment used to remeasure this relative dose, recheck the calculated dose. If the relative dose difference is still larger than the tolerance values, the authors will recommission this TPS.

\subsection{Relative Dose}

The calculated profile and measured profile are plotted in one figure as Fig. 2.

After analysis process of all profile line and percent depth dose line of each field size, the differences between calculated results and measured results are listed in Tables 2 and 3.

\subsection{Absolute Dose Results}

If the absolute dose difference is larger than the tolerance values, the authors will recheck method, set-up and equipment, all correction factor using to

Table 1 The limitations of difference between the calculated dose and measured dose.

\begin{tabular}{lllll}
\hline Situation & Square fields & Rectangular fields & Asymmetric fields & SSD variations \\
\hline Tolerance [\%] & 0.5 & 0.5 & 1 & 1 \\
\hline
\end{tabular}
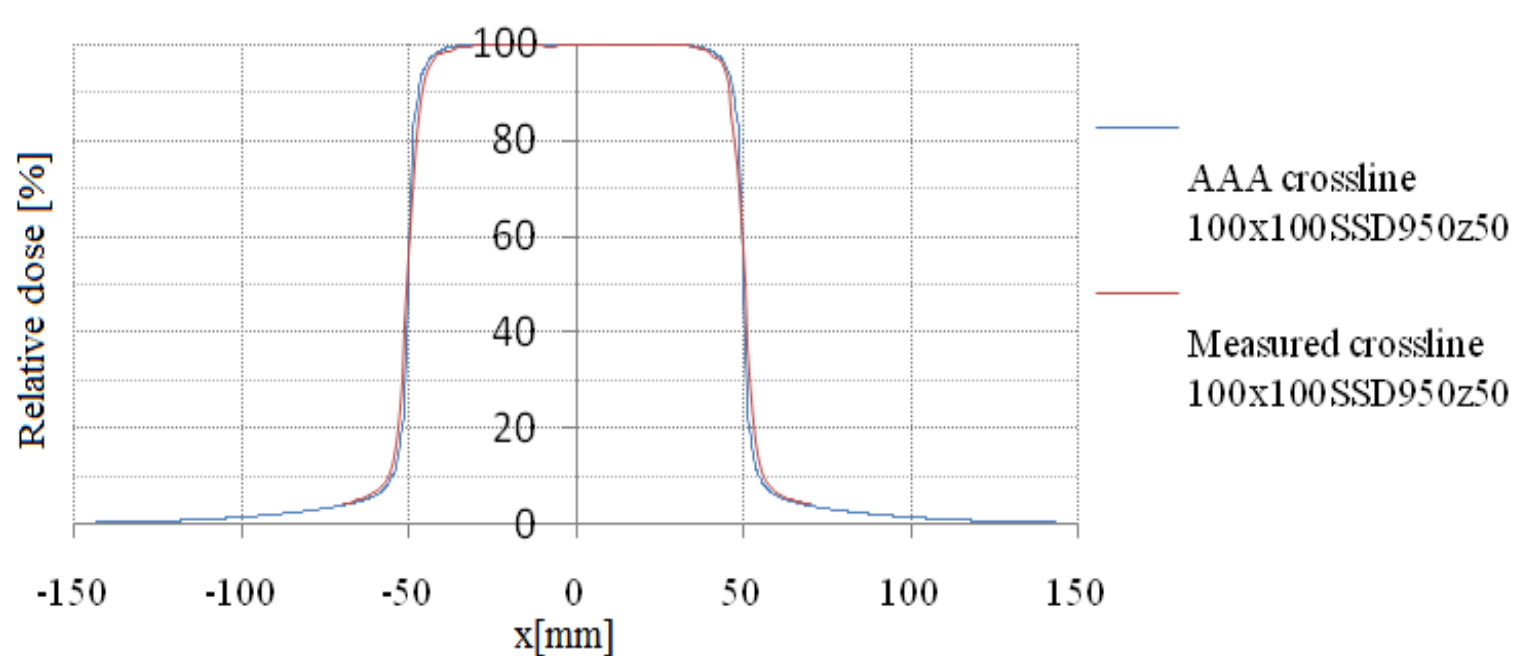

Fig. 2 The calculated crossline and the measured crossline at $50 \mathrm{~mm}$ depth $100 \mathrm{~mm}$ x $10 \mathrm{~mm}$ field size and $950 \mathrm{~mm}$ SSD. 

New Treatment Planning System

Table 2 The difference between the calculated profile and measured profile.

\begin{tabular}{|c|c|c|c|c|c|c|c|c|}
\hline \multirow{2}{*}{ Parameters } & \multirow{2}{*}{$\frac{\text { Field }}{[\mathrm{mm} \times \mathrm{mm}]}$} & \multirow{2}{*}{$\begin{array}{l}\text { SSD } \\
{[\mathrm{mm}]}\end{array}$} & \multicolumn{2}{|l|}{ Depth $d_{\max }$} & \multicolumn{2}{|c|}{ Depth $50 \mathrm{~mm}$} & \multicolumn{2}{|c|}{ Depth $150 \mathrm{~mm}$} \\
\hline & & & Crossline & Inline & Crossline & Inline & Crossline & Inline \\
\hline \multicolumn{9}{|c|}{ Limited value $\delta_{1}: 2 \%$} \\
\hline \multirow{11}{*}{$\delta_{1}[\%]$} & $100 \times 100$ & 950 & -0.2 & 0.1 & -0.5 & 0.1 & & \\
\hline & $100 \times 100$ & 850 & 0.8 & -0.3 & 0.5 & -0.3 & 0.4 & -0.3 \\
\hline & $100 \times 100$ & 1,400 & -0.5 & 0.2 & 0.2 & -0.5 & & \\
\hline & $70 \times 70$ & \multirow{8}{*}{950} & 0.2 & 0.7 & -0.1 & -0.4 & & \\
\hline & $230 \times 230$ & & 1.2 & -0.5 & -1.8 & 1.2 & & \\
\hline & $250 \times 250$ & & -0.4 & -0.5 & 1 & 1.1 & & \\
\hline & $30 \times 300$ & & -0.2 & 1 & -0.5 & -1.2 & & \\
\hline & $300 \times 30$ & & 1.5 & 0.2 & 0.2 & 0.3 & & \\
\hline & offset 1 & & 0.6 & 0.2 & 0.6 & 0.4 & & \\
\hline & offset 2 & & -0.2 & -0.4 & -0.5 & 2 & & \\
\hline & offset 3 & & 0.6 & -0.4 & 0.5 & 0.3 & & \\
\hline \multicolumn{9}{|c|}{ Limited value $\delta 2: 2 \mathrm{~mm}$} \\
\hline \multirow{11}{*}{$\delta_{2}[\mathrm{~mm}]$} & $100 \times 100$ & 950 & 0.7 & -0.4 & 1.8 & 0.6 & & \\
\hline & $100 \times 100$ & 850 & 1.6 & 1.2 & 1.6 & -0.5 & 1.4 & -0.5 \\
\hline & $100 \times 100$ & 1,400 & 1.8 & 1.1 & 2.0 & -0.2 & & \\
\hline & $70 \times 70$ & \multirow{8}{*}{950} & -1.0 & -1.0 & -1.5 & 0.4 & & \\
\hline & $230 \times 230$ & & 0.2 & -0.2 & -0.5 & -0.3 & & \\
\hline & $250 \times 250$ & & 1.3 & 0.5 & 1.0 & -0.4 & & \\
\hline & $30 \times 300$ & & 1.5 & 0.5 & -2.0 & -0.5 & & \\
\hline & $300 \times 30$ & & 1.5 & 2 & -1.3 & 1.8 & & \\
\hline & Offset 1 & & 0.3 & 0.5 & 0.6 & 0.5 & & \\
\hline & Offset 2 & & 1.5 & -0.5 & 1.0 & -1.0 & & \\
\hline & Offset 3 & & 1.5 & -0.8 & 1.5 & -1.0 & & \\
\hline \multicolumn{9}{|c|}{ Limited value $\delta_{3}: 3 \%$} \\
\hline \multirow{11}{*}{$\delta_{3}[\%]$} & $100 \times 100$ & 950 & 1.5 & 2.5 & 2.5 & -1.0 & & \\
\hline & $100 \times 100$ & 850 & 2.0 & 2.3 & 2.2 & -1.0 & 2.0 & 0.9 \\
\hline & $100 \times 100$ & 1,400 & -0.9 & 3.0 & -1.5 & 3.0 & & \\
\hline & $70 \times 70$ & \multirow{8}{*}{950} & -1.0 & -2.0 & 2.6 & -3.0 & & \\
\hline & $230 \times 230$ & & 1.0 & -2.4 & 0.4 & -2.5 & & \\
\hline & $250 \times 250$ & & 2.3 & -2.2 & -2.0 & 1.5 & & \\
\hline & $30 \times 300$ & & -2.0 & -1.0 & 2.5 & -1.5 & & \\
\hline & $300 \times 30$ & & 1.3 & -2.5 & 3.0 & 1.3 & & \\
\hline & Offset 1 & & 2.6 & 1.5 & -2.4 & 2.6 & & \\
\hline & Offset 2 & & -1.5 & -2.0 & 2.8 & -1.5 & & \\
\hline & Offset 3 & & -2.0 & -3.0 & -2.2 & 0.9 & & \\
\hline \multicolumn{9}{|c|}{ Limited value $\delta_{4}: 3 \%$} \\
\hline \multirow{11}{*}{$\delta_{4}[\%]$} & $100 \times 100$ & 950 & 2.0 & -0.5 & 1.5 & -1.0 & & \\
\hline & $100 \times 100$ & 850 & 2.0 & 1.0 & 1.0 & 2.0 & 1.0 & 2.0 \\
\hline & $100 \times 100$ & 1,400 & 2.0 & 2.3 & 0.5 & -2.0 & & \\
\hline & $70 \times 70$ & \multirow{8}{*}{950} & -1.5 & -2.5 & 3.0 & 2.5 & & \\
\hline & $230 \times 230$ & & 0.4 & 0.5 & -0.8 & 0.5 & & \\
\hline & $250 \times 250$ & & -2.0 & 0.3 & 1.5 & 0.5 & & \\
\hline & $30 \times 300$ & & 2.5 & -1.5 & -2.0 & -1.5 & & \\
\hline & $300 \times 30$ & & 2.7 & -1.0 & -3.0 & -1.2 & & \\
\hline & Offset 1 & & -2.5 & 0.5 & 2.5 & 0.5 & & \\
\hline & Offset 2 & & 1.6 & 2.0 & 2.2 & 2.0 & & \\
\hline & Offset 3 & & -2.6 & 3.0 & -1.5 & 2.0 & & \\
\hline
\end{tabular}



New Treatment Planning System

Table 3 The difference between the calculated PDD and measured PDD.

\begin{tabular}{llll}
\hline $\begin{array}{l}\text { Field size } \\
{[\mathrm{mm} \times \mathrm{mm}]}\end{array}$ & $\begin{array}{l}\text { SSD } \\
{[\mathrm{mm}]}\end{array}$ & $\delta_{1}[\%]$ & $\delta_{2}[\mathrm{~mm}]$ \\
\hline $100 \times 100$ & 850 & -0.38 & 0.4 \\
$100 \times 100$ & 950 & 0.53 & 0.3 \\
$100 \times 100$ & 1,400 & -1.4 & 2.0 \\
$70 \times 70$ & & 0.91 & 0.9 \\
$230 \times 230$ & 950 & -1.95 & 1.0 \\
$250 \times 250$ & & 0.54 & 0.8 \\
$30 \times 300$ & & 0.49 & 0.4 \\
$300 \times 30$ & 0.92 & 1.2 \\
Offset 1 & & 0.81 & 1.3 \\
Offset 2 & & 0.55 & 0.8 \\
Offset 3 & & 1.24 & 1.3 \\
Limited value & & $2 \%$ & $2 \mathrm{~mm}$ \\
\hline
\end{tabular}

Table 4 The difference between absolute doses at center of fields, $50 \mathrm{~mm}$ depth, which are calculated with new TPS, and measured absolute dose

\begin{tabular}{llllllllll}
\hline $\begin{array}{l}\text { Field size } \\
{[\mathrm{mm} \times \mathrm{mm}]}\end{array}$ & $\begin{array}{l}\text { SSD } \\
{[\mathrm{mm}]}\end{array}$ & \multirow{2}{*}{ MU } & $\begin{array}{l}\text { Mea.1 } \\
{[\mathrm{cGy}]}\end{array}$ & $\begin{array}{l}\text { Mea.2 } \\
{[\mathrm{cGy}]}\end{array}$ & $\begin{array}{l}\text { Mea.3 } \\
{[\mathrm{cGy}]}\end{array}$ & $\begin{array}{l}\text { Mean } \\
{[\mathrm{cGy}]}\end{array}$ & $\begin{array}{l}\text { Calculated } \\
{[\mathrm{cGy}]}\end{array}$ & $\Delta[\%]$ & $\begin{array}{l}\text { Limited value } \\
{[\%]}\end{array}$ \\
\hline $100 \times 100$ & 850 & \multirow{2}{*}{100} & 116.2 & 116.0 & 116.3 & 116.2 & 116.2 & -0.03 & 1 \\
$100 \times 100$ & 950 & & 94.6 & 94.5 & 94.5 & 94.5 & 94.4 & 0.1 & \\
$100 \times 100$ & 1,400 & & 45.4 & 45.4 & 45.4 & 45.4 & 45.6 & -0.05 & 1 \\
$70 \times 70$ & & & 91.4 & 91.4 & 91.5 & 91.4 & 91.1 & 0.37 & \\
$230 \times 230$ & & 100.8 & 100.8 & 100.9 & 100.8 & 101.1 & -0.26 & 0,5 \\
$250 \times 250$ & & 101.6 & 101.6 & 101.4 & 101.5 & 101.7 & -0.16 & & \\
$30 \times 300$ & \multirow{2}{*}{100} & 88.9 & 89 & 89.1 & 89 & 88.7 & 0.34 & 0.5 \\
$300 \times 30$ & & 87.8 & 87.8 & 87.8 & 87.8 & 87.6 & 0.23 & & \\
Offset 1 & & & 86.2 & 85.9 & 86.1 & 86.1 & 85.8 & 0.31 & \\
Offset 2 & & 86.1 & 86.1 & 86.1 & 86.1 & 85.8 & 0.35 & 1 \\
Offset 3 & & 86 & 86.1 & 86.3 & 86.1 & 85.8 & 0.39 & \\
\hline
\end{tabular}

remeasure this absolute dose, recheck the calculated dose. If the absolute dose difference is still larger than the tolerance values, the authors will recommission this TPS.

The difference between absolute doses at center of fields, $50 \mathrm{~mm}$ depth, which are calculated with new TPS, and measured absolute dose, are listed in Table 4.

\subsection{Quality Assurance IMRT Plan Results}

Statistic of the difference between calculated dose at center of PTV, spinal cord, DHP, DHT, MTP, MTT and the measured dose of the fifty head and neck cancer patients is plotted in Figs. 3-8.

\section{Discussion}

Comparing relative dose results, it includes the profiles and PDD, with limited difference in IAEA No. 430 , it shows that the differences between calculated relative dose of interpolated field in water phantom with measured relative doses in water phantom are smaller than the difference standard values.

Compared absolute dose results, it shows that the differences between calculated absolute dose at centre of interpolated field in water phantom with measured absolute doses are also smaller than the difference standard values for absolute doses.

Mean difference between measured dose and calculated dose at center of PTV, center of spinal cord, right submandibular gland, left submandibular, right parotid gland, left parotid gland are 0.197; -0.414; $0.071 ;-0.25 ; 1.883 ;-1.024$ respectively. All the different values of centre DHP, DHT, MTP, MTT are 


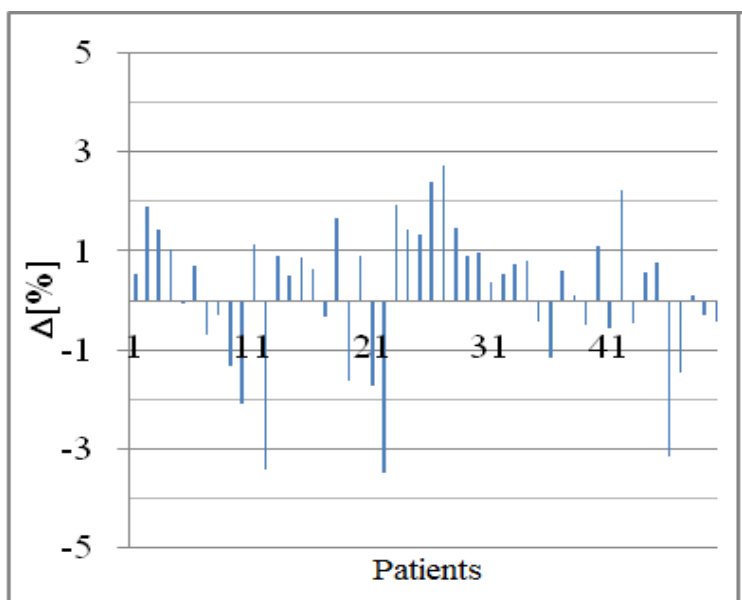

Fig. 3 The difference $(\Delta \%)$ between measured dose and calculated dose at center of PTV of fifty patients.

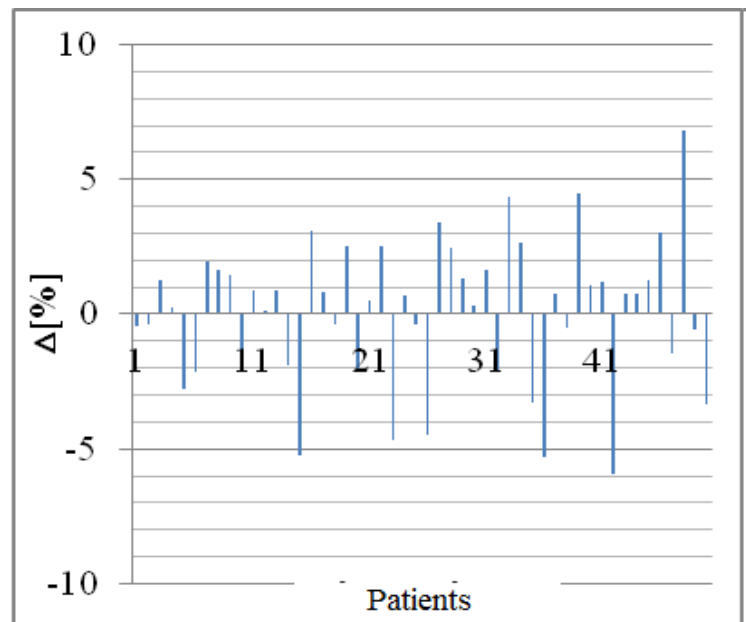

Fig. 5 The difference $(\Delta \%)$ between measured dose and calculated dose at center of DHP of fifty patients.

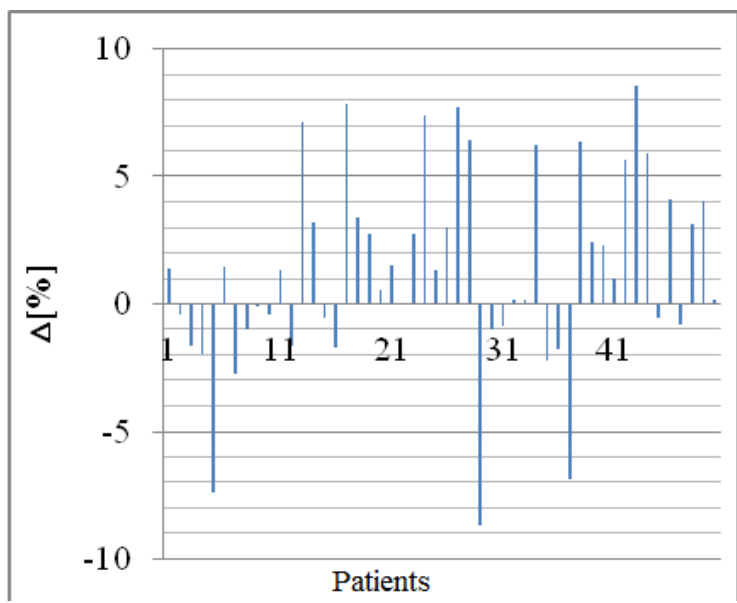

Fig. 7 The difference $(\Delta \%)$ between measured dose and calculated dose at center of MTP of fifty patients.

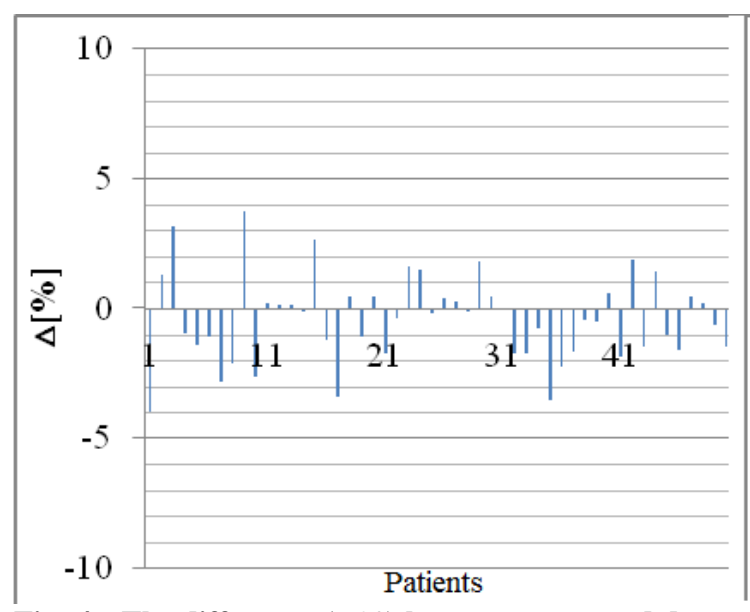

Fig. 4 The difference $(\Delta \%)$ between measured dose and calculated dose at center of spinal cord of fifty patients.

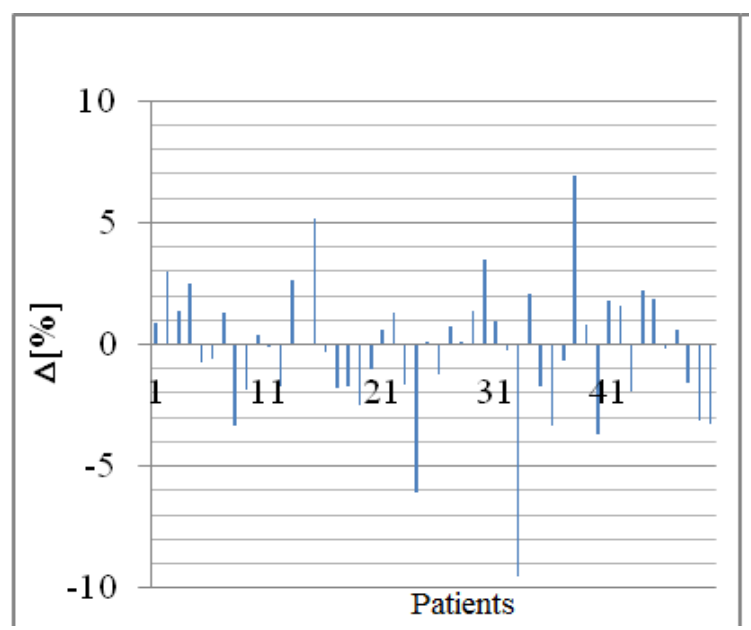

Fig. 6 The difference $(\Delta \%)$ between measured dose and calculated dose at center of DHT of fifty patients.

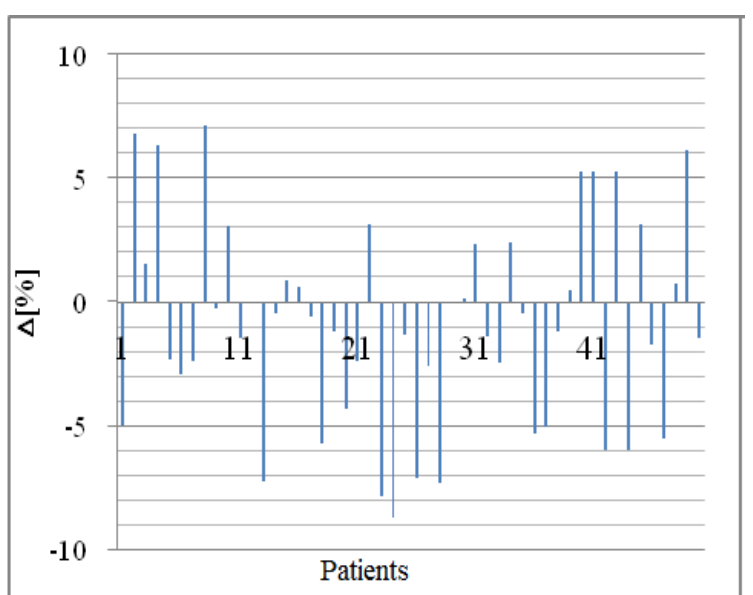

Fig. 8 The difference $(\Delta \%)$ between measured dose and calculated dose at center of MTT of fifty patients. 
less than $10 \%$. The results are fitting with guideline in ESTRO Booklet No. 9 when using FC 65 for quality controlled IMRT plan [6,7].

\section{Conclusions}

The accuracy calculated doses with AAA algorithm in new TPS match the standards in IAEA No. 430.

Some situations FC 65 in plastic phantom are used to control quality of IMRT plan, however, it must accept the difference between calculated dose and measured dose until $10 \%$.

\section{References}

[1] IAEA Technical Reports Series No. 430. Chapter 9 Commissioning “Commissioning and Quality Assurance of Computerized Planning Systems for Radiation Treatment of Cancer.” IAEAL 04-00363.

[2] Associazione Italiana Fisica Medica AIFM Report AIFM. 2011. "protocollo per il controllo della qualità dei sistemi per pianificazione radioterapica (RTPS). No. 7.

[3] IAEA-TECDOC-1583. "Commissioning of Radiotherapy Treatment Planning Systems: Testing for Typical External Beam Treatment Techniques.” ISBN 978-92-0-100508-3. ISSN 1011-4289.

[4] Khan, F. M. Treatment Planning in Radiation Oncology. 2nd ed.

[5] Dyk, H. V., Barnett, R. B., and Battista, J. J. "Chapter 8: Computerized Radiation Treatment Planning Systems.” Modern Technology of Radiation Oncology.

[6] VARIAN Medical Systems. "Eclipse Algorithms Reference Guide.” P/N B5021612RO1A. AUGUST 2009.

[7] Albber, M., Broggi, S., Wagter, C. D., Eichwurzel, I., Engstrom, P., and Fiorino, C. 2008. "ESTRO Booklet No. 9: Guidelines for the Verification of IMRT.” First edition IBN 90-804532-9 (C) 2008 by ESTRO.

[8] IAEA Technical reports series No. 398. “Absorbed Dose Determination in External Beam Radiotherapy An International Code of Practice for Dosimetry Based on Standards of Absorbed Dose to Water.” International Atomic Energy Agency, Vienna. 